OPEN ACCESS

Edited by:

Massimo Lucarini,

Council for Agricultural and

Economics Research, Italy

Reviewed by:

Palanivel Ganesan,

Konkuk University, South Korea

Uma Devi Palanisamy,

Monash University Malaysia, Malaysia

${ }^{*}$ Correspondence:

Hock Eng Khoo

hockeng_khoo@yahoo.com

Azrina Azlan

azrinaaz@upm.edu.my

Specialty section:

This article was submitted to

Food Chemistry,

a section of the journal

Frontiers in Chemistry

Received: 07 May 2018 Accepted: 07 January 2019 Published: 31 January 2019

Citation:

Khoo HE, Azlan A and Abd Kadir NAA (2019) Fatty Acid Profile,

Phytochemicals, and Other

Substances in Canarium

odontophyllum Fat Extracted Using

Supercritical Carbon Dioxide.

Front. Chem. 7:5.

doi: 10.3389/fchem.2019.00005

\section{Fatty Acid Profile, Phytochemicals, and Other Substances in Canarium odontophyllum Fat Extracted Using Supercritical Carbon Dioxide}

\author{
Hock Eng Khoo ${ }^{1,2 *}$, Azrina Azlan ${ }^{1,2 *}$ and Noor Atiqah Aizan Abd Kadir ${ }^{1}$ \\ ${ }^{1}$ Department of Nutrition and Dietetics, Faculty of Medicine and Health Sciences, Universiti Putra Malaysia, UPM Serdang, \\ Malaysia, ${ }^{2}$ Research Centre of Excellence for Nutrition and Non-Communicable Diseases, Faculty of Medicine and Health \\ Sciences, Universiti Putra Malaysia, UPM Serdang, Malaysia
}

This study aims to identify potential phenolic compounds, terpenoids, and other phytochemicals, as well as fatty acid profile and peptides in Canarium odontophyllum (CO) oil and oleoresin, extracted using supercritical carbon dioxide. LC-ESI-MS was applied in separation and tentative identification of phytochemicals in $\mathrm{CO}$ oil and oleoresin. Based on the results, 11 common fatty acids and their isomers, monoglycerides, diglycerides, as well as other types of lipid, were tentatively identified in the $\mathrm{CO}$ oil and oleoresin. The identified fatty acids consisted of saturated fatty acids (C8-C16), monounsaturated fatty acids (C16:1 and C18:1), polyunsaturated fatty acids (C18:2, C18:3, C18:4, and C20:3), and other unclassified fatty acids. The tentatively identified phenolic compounds were phenolic acids, flavonoids, lignans, and a phenolic monoester. Triterpenes, sesquiterpenes, and apocarotenoids were the terpenoids found in $\mathrm{CO}$ oil and oleoresin. Besides these typical bioactives, some volatiles, aromatic compounds, peptides, and other known and unknown phytochemicals were also tentatively identified in the oil and oleoresin of CO. Some of these compounds are new compounds identified in $\mathrm{CO}$ oil and oleoresin, which are not found in many other fruit oils. Although $\mathrm{CO}$ oil and oleoresin contain a small number of phytochemicals, their contribution as antioxidants may prevent several diseases. In this study, we hypothesized that $\mathrm{CO}$ oleoresin contains certain types of fatty acids that render its semi-solid together with other chemical components which are not found in $\mathrm{CO}$ oil. This is the first study that tentatively identified fatty acids, peptides, and potential phytochemicals in $\mathrm{CO}$ oil and oleoresin using LC-ESI-MS.

Keywords: dabai, LCMS, oil, oleoresin, peptide, terpenoid, volatile

\section{INTRODUCTION}

The Canarium odontophyllum Miq. fruit, also known as dabai, is one of the species of Canarium fruits from the Burseraceae family that exists in the Southeast Asian region. The trees of Canarium odontophyllum (CO) are largely cultivated in the Borneo region, such as the states of Sarawak and Sabah. Physical description of dabai has been reported previously; the fruit is usually dark purple to black in color, and it has oily pulp. CO fruit is $3.7-4.1 \mathrm{~cm}$ in length and $2.4-2.8 \mathrm{~cm}$ in width, with a hard 3-angular-seed (Khoo, 2014). 
Nutritional values and antioxidant properties of the CO fruit have been reported in our previous studies. The CO pulp has a high oil content (Chew et al., 2011). The fat content of CO fruit ranged between $21.16 \pm 4.71$ and $25.76 \pm 3.03 \mathrm{~g} / 100 \mathrm{~g}$ fresh weight. According to Azlan et al. (2010), the CO pulp contained $44.4 \%$ total saturated fats, $42.8 \%$ total monounsaturated fats, and $12.8 \%$ total polyunsaturated fats. Besides total fatty acids, the study also reported that the $\mathrm{CO}$ oil had, as the main fatty acids, $36.1 \%$ palmitic acid, 5.8\% myristic acid, $41.5 \%$ oleic acid, and $11.8 \%$ linoleic acid. In addition to the balanced fatty acid composition, $\mathrm{CO}$ pulp had moisture, total available carbohydrate, protein, and ash content of 50.4-51.9\%, 4.5-9.2\%, 3.5-5.2\%, and $1.7-1.9 \%$, respectively; mineral content, amino acid composition, as well as antioxidative properties, were also determined in the CO pulp (Chew et al., 2011).

The extracts of defatted CO pulp have been shown to possess antioxidative properties and cardioprotective effects (Khoo et al., 2014). The defatted CO pulp contained anthocyanins as the major antioxidants. The anthocyanin-rich defatted CO pulp significantly reduced plasma total cholesterol and plasma LDLcholesterol of the hypercholesterolemic rabbits compared to the positive control (Shakirin et al., 2012b). Aqueous extract of CO leaves was also able to reduce high blood pressure through vasodilation of the rat thoracic aorta (Basri et al., 2018).

The balanced fatty acid composition in CO oil demonstrated a protective effect in healthy rabbits (Shakirin et al., 2012a). In the study, Shakirin et al. (2012a) reported that CO oil brought about a significant improvement in lipid profile (increased plasma HDLcholesterol, and reduced plasma LDL-cholesterol and triglyceride levels), as well as reduced TBARS (thiobarbituric acid reactive substances) level and favorable changes in the levels of plasma malonaldehyde, superoxide dismutase, glutathione peroxidase, and total antioxidant status. The oil had a total phenolic content of $20.27 \mathrm{mg}$ gallic acid equivalent/100 $\mathrm{g}$ oil. The high amount of phenolic compounds determined in CO pulp oil could contribute to the protective effect of the fruit in vivo.

Extraction method and type of solvent used are essential for extracting nutrients and phytochemicals from a high-fat fruit. As dabai is a high-fat fruit, the fruit pulp contains both polar and non-polar phytochemicals. Although methanol has been recognized as the widely used solvent for extraction and isolation of chemical components from plants, it is known to be toxic to the human body if ingested in even a small amount. The use of greener solvents such as water and carbon dioxide is recommended. However, water is a polar solvent, and it is hydrophilic. For that reason, supercritical carbon dioxide $\left(\mathrm{SCO}_{2}\right)$ is the best to be used for extracting nutrients and phytochemicals with a wide range of polarity from any sources (Mukhopadhyay, 2009). $\mathrm{CO}_{2}$ is also non-toxic and recyclable. The solubility behavior of the non-polar phytochemicals in $\mathrm{SCO}_{2}$ has been reported in a previous study (Güçlü-Üstündag and Temelli, 2004). The study reported that the solubility of the compounds is dependent on the operating conditions. The use of an optimized extracting condition warrants optimal extraction yield with more types of chemical components isolated from a plant sample.

So far, except for the proximate analyzes and antioxidant properties and the determination of fatty acid and amino acid profile, as well as selected vitamins and minerals, limited studies have been done on the chemical analysis of $\mathrm{CO}$ oil. There has been no report on the chemical properties of $\mathrm{SCO}_{2}$ extracted CO oil and oleoresin. Due to the lack of data for the complete identification of phytochemicals or plant metabolites and nutritional components in $\mathrm{SCO}_{2}$ extracted $\mathrm{CO}$ oil and oleoresin, there is a need to characterize and to identify potential chemical components in the fat samples. This study was also performed to identify fatty acids, peptides, and potential phytochemicals in CO oil and oleoresin using LC-ESI-MS technique, as well as to quantify the fatty acid composition in the oil and oleoresin. Moreover, this is the first study on the qualitative analysis of chemical components in $\mathrm{CO}$ oil and oleoresin.

\section{MATERIALS AND METHODS Chemicals and Reagents}

Acetonitrile of LiChrosolv grade and methanol used in the fractionation of phytochemicals, peptides, and fatty acids were purchased from Merck (Darmstadt, Germany). Water was purified by a Milli-Q system from Millipore (Milford, USA).

\section{Preparation of Sample and Extraction}

The CO fruit $(200 \mathrm{~kg}$ ) was purchased from an identified supplier through Semongok Agriculture Research Centre, Sarawak. About $100 \mathrm{~kg}$ of blanched $\mathrm{CO}$ pulp (at $60^{\circ} \mathrm{C}$ for $15 \mathrm{~min}$ ) was obtained upon removal of the kernel. The CO pulp was freeze-dried using a benchtop freeze-drier (Virtis, NY, USA), and the lyophilized pulp was pulverized using a household blender (Panasonic, Malaysia) and sieved by a 20-mesh plastic sieve. Lipids from the lyophilized samples were extracted using an ABRP200 $\mathrm{SCO}_{2}$ extractor (Thar Process, PA, USA).

We have previously optimized the $\mathrm{SCO}_{2}$ extraction conditions and established a laboratory protocol for the $\mathrm{CO}$ fat extraction (Jelani et al., 2017). However, the extraction of CO fat was done based on the semi-pilot scale, and the optimized extraction condition was applied. In this study, CO fat was extracted from the $\mathrm{CO}$ pulp powder using the $\mathrm{SCO}_{2}$ extractor. The extraction was done by the automated system with the heater set to $40^{\circ} \mathrm{C}$ and with an extraction pressure of $40 \mathrm{MPa}$.

The extraction of $\mathrm{CO}$ oil was done using the extractor with a volume of $500 \mathrm{ml}$. Before the injection of $\mathrm{CO}_{2}$ into the extraction vessel, $50 \mathrm{~kg}$ of the lyophilized sample was inserted into the vessel. The extraction was done based on the optimized conditions for CO oil extraction as follows: an extraction pressure of $40 \mathrm{MPa}$ and an extraction temperature of $40^{\circ} \mathrm{C}$. The flow of $\mathrm{SCO}_{2}$ was maintained at $15 \mathrm{~g} / \mathrm{min}$, and the static duration was set to $30 \mathrm{~min}$. The CO pulp powder was soaked in the vessel containing the $\mathrm{SCO}_{2}$ at high pressure for $30 \mathrm{~min}$. After $30 \mathrm{~min}$ of soaking, the oleoresin and oil were removed from the separation vessel via a manual valve after the extraction was completed. The extraction process took a total of $2 \mathrm{~h}$. The extracted oil was collected as CO oil, and the semi-solid fraction was collected as CO oleoresin. Extraction of oil was performed in three replicates.

Semi-polar extracts of the collected oil and oleoresin were obtained by extraction with aqueous methanol. The extraction 


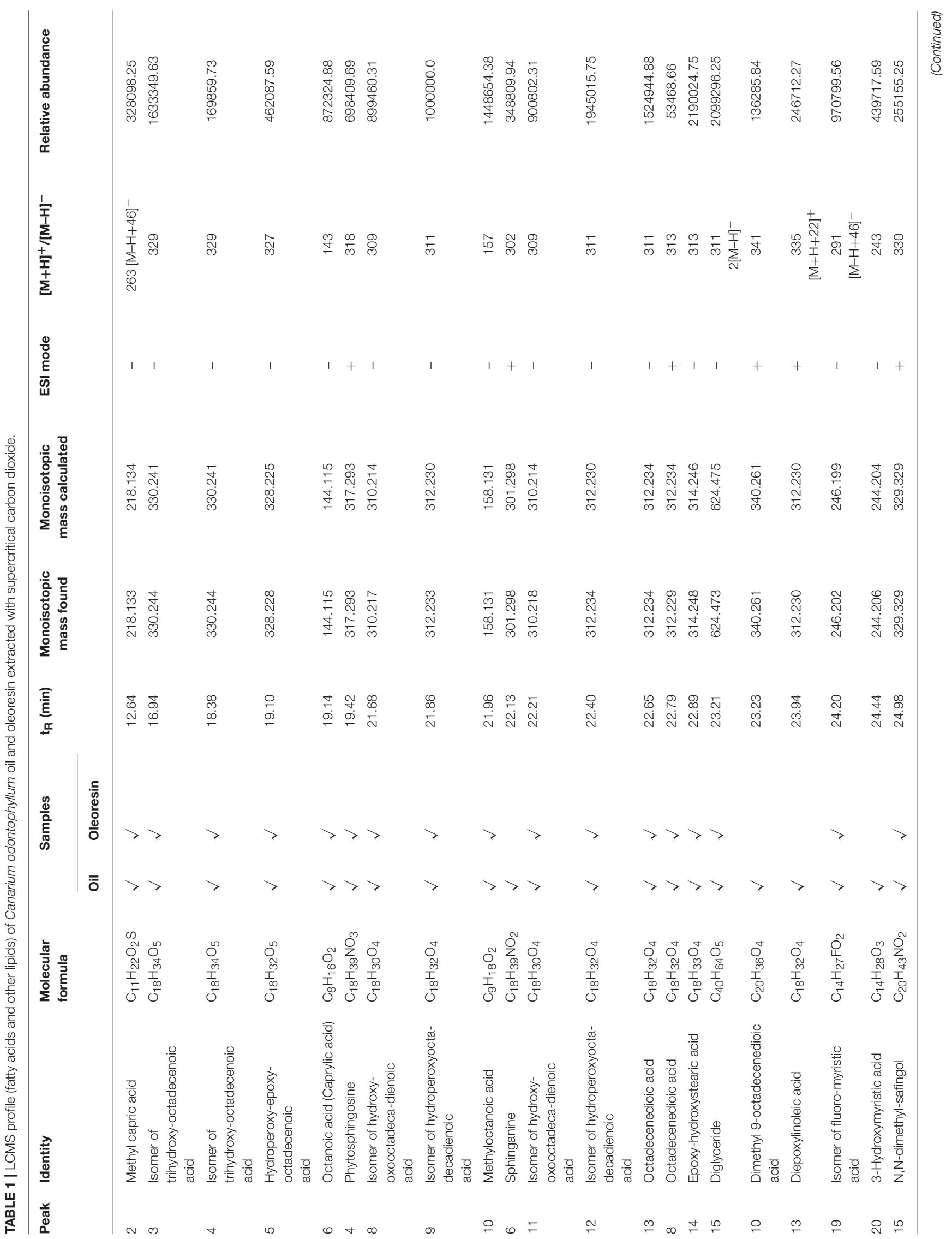




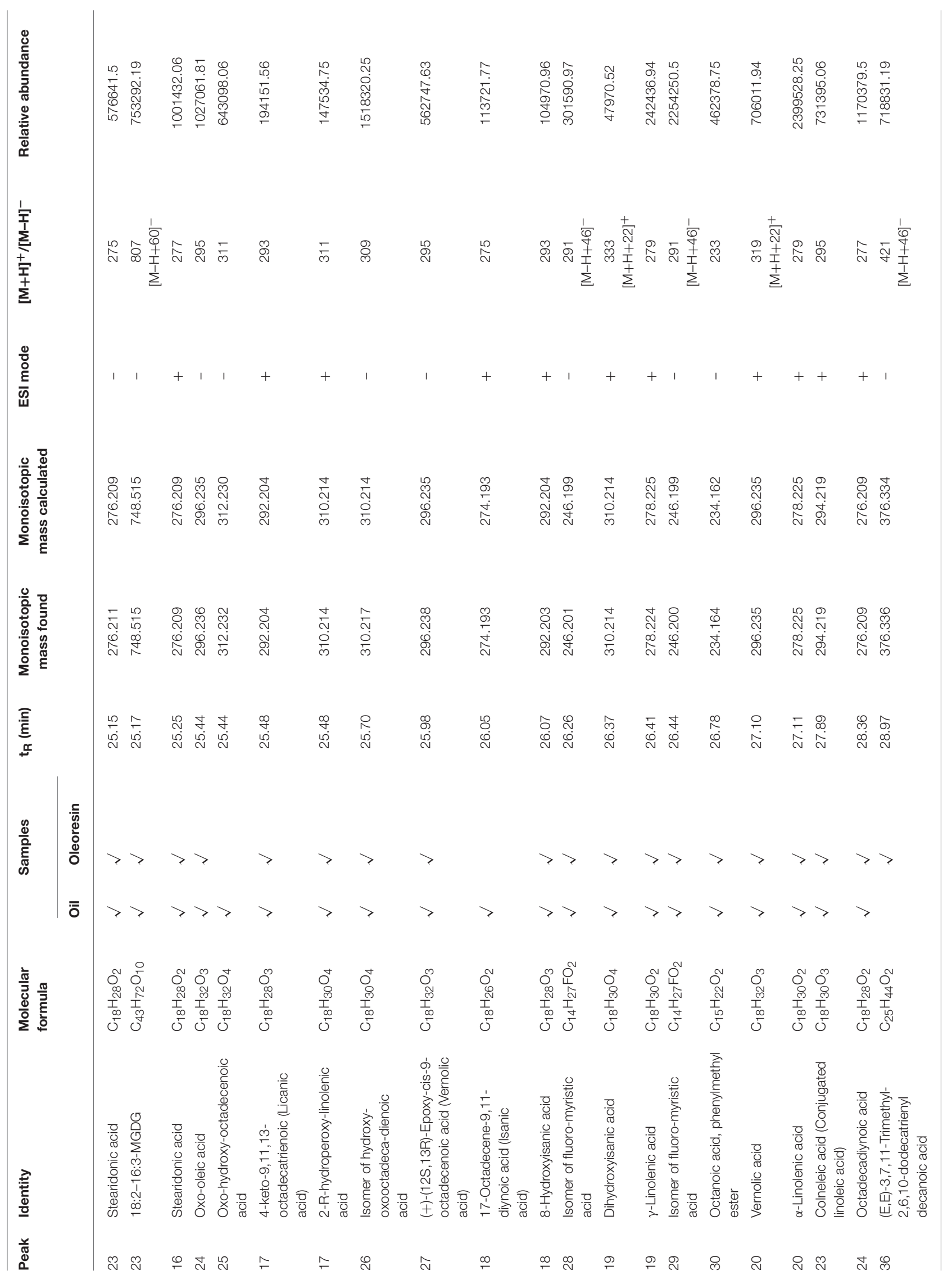




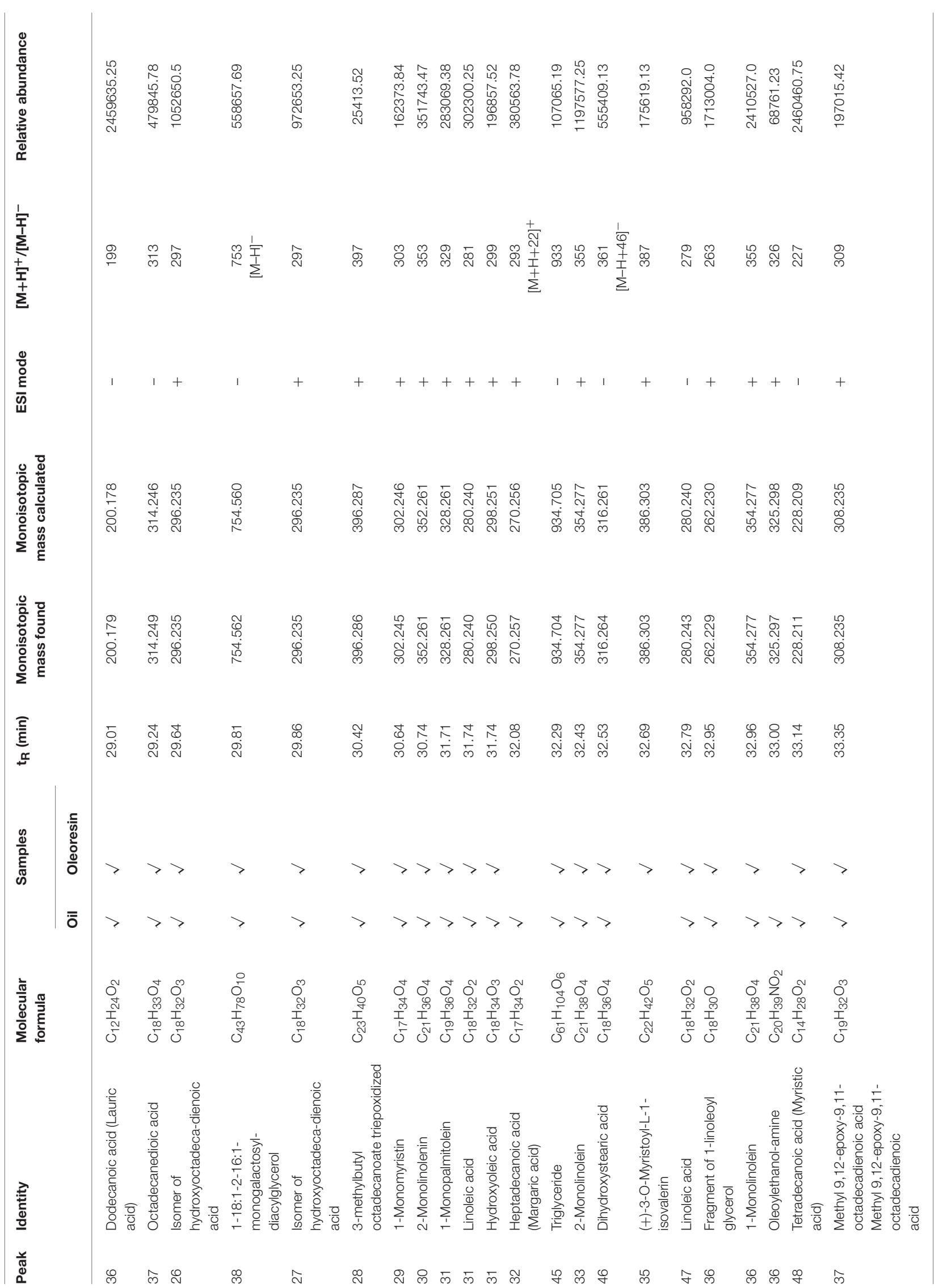




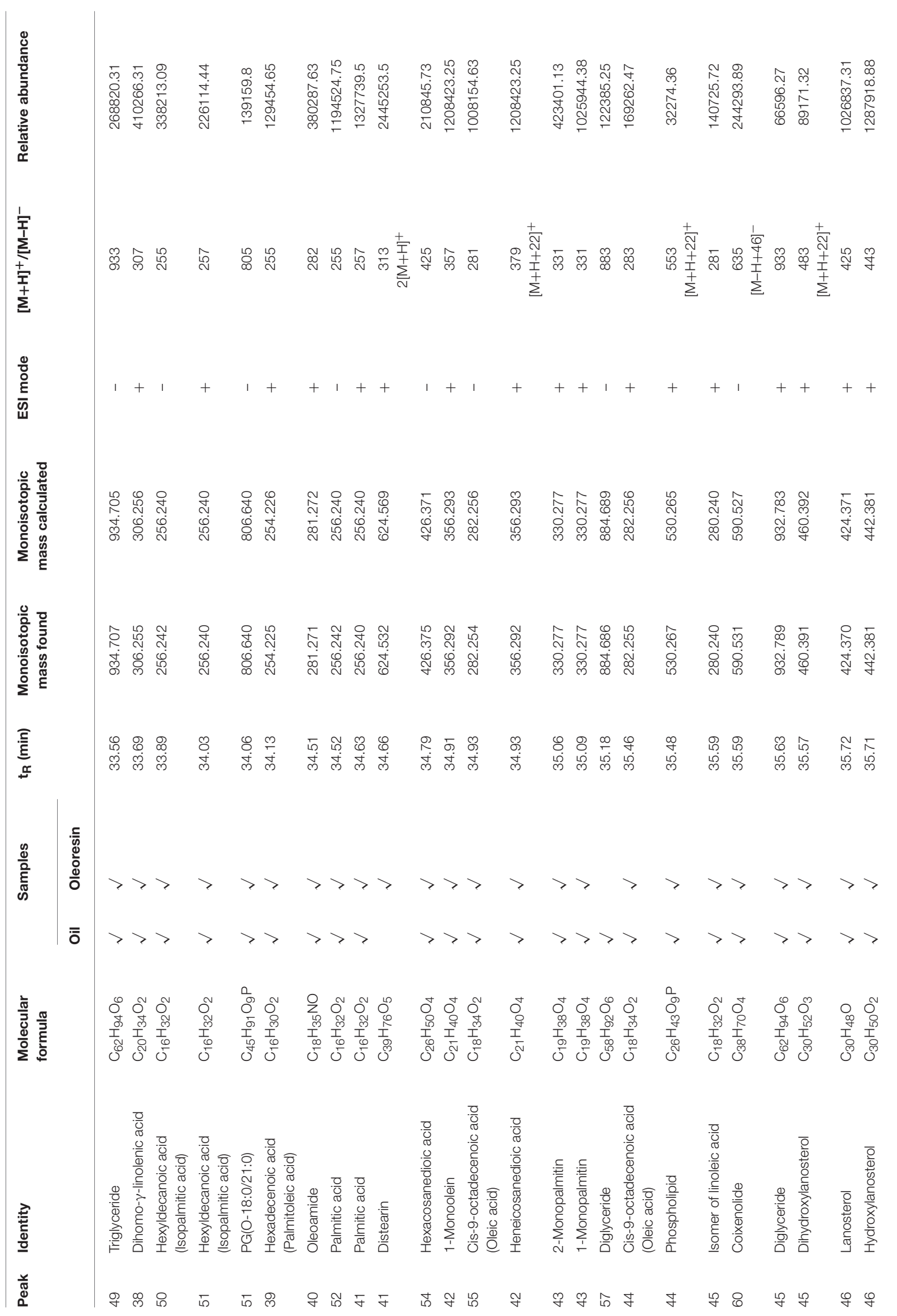


was previously optimized using several extraction solvents, although methanol was found to be the ideal extraction solvent for extracting phytochemicals from CO oil, especially polyphenols, terpenoids, and glycosides (Biswas et al., 2013). The chemical components of $\mathrm{CO}$ oil and oleoresin were extracted using aqueous $95 \%$ methanol $(1: 10 \mathrm{w} / \mathrm{v})$ at room temperature for $20 \mathrm{~min}$. The oil layer was separated using a separating funnel. The methanolic extracts were filtered using $0.45 \mathrm{~nm}$ Sartorius syringe filters before being subjected to liquid chromatography analysis. Triplicate extraction was performed on both samples.

\section{LC-ESI-MS Method}

An Agilent 1290 Liquid Chromatography system (Agilent Technologies, CA, USA) coupled with a 6520 Q-TOF tandem mass spectrometer was used to separate compounds from the methanolic extracts of $\mathrm{CO}$ oil and oleoresin. The mass detector was a Q-TOF accurate mass spectrometer equipped with an electrospray ionization (ESI) interface and controlled by the MassHunter software. In brief, $2 \mu$ l of the sample solution comprising a mixture of compounds were loaded on a $2.1 \mathrm{~mm}$ (I.D.) Agilent Zorbax Eclipse Plus C-18 (100 mm in length) analytical column (with a particle size of $1.8 \mu \mathrm{m}$ ) that was maintained at $25^{\circ} \mathrm{C}$. The analysis (positive mode) was performed by applying a gradient run that comprised mobile phase A $(0.1 \%$ formic acid in water) and mobile phase B (100\% acetonitrile with $0.1 \%$ formic acid), with a flow rate of $0.25 \mathrm{ml} / \mathrm{min}$. As for the negative mode, the mobile phases used were mobile phase $\mathrm{A}$ $(0.1 \%$ ammonium formate in water) and mobile phase B $(100 \%$ acetonitrile).

Several trials were conducted to optimize the chromatographic conditions prior to the actual analysis by the application of different compositions and gradients of mobile phases. The gradient was run as follows: $5-90 \%$ B for $35.0 \mathrm{~min}$, $90-90 \%$ B for $6 \mathrm{~min}, 90-5 \%$ for $0.1 \mathrm{~min}$, and $5-5 \%$ for $6.9 \mathrm{~min}$. The total run time for the LCMS analysis was $48 \mathrm{~min}$, with a 13-min post-run time. The ionization conditions were adjusted to a capillary temperature of $300^{\circ} \mathrm{C}$ and 4000 voltage. The nebulizer pressure was $45 \mathrm{psi}$, and the nitrogen flow rate was 10 $1 / \mathrm{min}$. All mass spectrometry data were recorded in both positive and negative ion modes. The acquisition rate was $1.03 \mathrm{spectra} / \mathrm{s}$ across the ranges of $100-1000 \mathrm{~m} / z$ for both positive and negative ion modes.

Both CO samples were subjected to the LCMS Q-TOF in positive and negative ion modes for the profiling of the compounds or metabolites present in $\mathrm{CO}$ oil and oleoresin based on one replicate. Initially, the available plant metabolites were tentatively identified through the matching of masses of the compounds with the PlantCyc database. The MS mass tolerances were set between 0.05 and $0.1 \mathrm{Da}$. All the exact masses of unknown metabolites were further compared with the other online chemical databases (PubChem, LIPID MAPS, and ChemSpider), as well as some published scientific articles. The analysis was only focused on the electron ionization MS ranged between $m / z 100$ and $m / z 1,000$. The LCMS analysis was performed by the Proficient Lab Malaysia (Selangor, Malaysia) and the tentative identification of the compounds in the methanolic extracts of $\mathrm{CO}$ fat samples was done with the help of a chemist from the company.

\section{Fatty Acid Methyl Esters}

Analysis of fatty acid methyl esters (FAMEs) was performed based on the IUPAC 2.301 protocol (IUPAC, 1987). Briefly, FAMEs were analyzed and determined using the Waters Gas Chromatography (GC) coupled with a flame ionization detector. The GC column used was Agilent DB-23 Capillary GC Column, $60 \mathrm{~m} \times 0.25 \mathrm{~mm}$, id $0.25 \mu \mathrm{m}$ (J\&W Scientific, Inc., CA, USA). The temperatures for injector and detector were 130 and $220^{\circ} \mathrm{C}$, respectively. The nitrogen carrier gas flow was set at $0.53 \mathrm{ml} / \mathrm{min}$. Fatty acid content in $\mathrm{CO}$ oil and oleoresin was quantified and determined based on the FAMEs.

\section{RESULTS AND DISCUSSION}

\section{LCMS Profiling and Tentative Identification of Potential Compounds}

A sensitive, accurate, and specific method using highperformance liquid chromatography (HPLC) and ESI-MS was developed for the separation and tentative identification of compounds found in the semi-polar fraction (which consists of polar and non-polar compounds) of the fat samples. The molecular masses were assigned by ESI-MS. The subsequent structural characterization was carried out by a tandem mass spectrometric method. The accurate mass and fragmentation behavior of the compounds were investigated using ESI-Q-TOF in negative and positive ion modes.

Liquid chromatography-mass spectrometry (LCMS) combines the techniques of physical separation capabilities of liquid chromatography with mass analysis capabilities of mass spectrometry. This application is oriented toward the general detection and tentative identification of potential compounds in a plant mixture (Khoo et al., 2012). MS measures the massto-charge ratio of charged particles. It is used for determining masses of particles and the elemental composition of a sample or molecule, as well as for elucidating the chemical structure of molecules. Fatty acids, lipid metabolites, phenolic compounds, terpenoids, peptides, and other chemical components in the extracts of CO fat samples were tentatively identified based on the available plant metabolites and chemical databases.

\section{Fatty Acids and Other Lipids}

As shown in Table 1, the identified fatty acids in CO oil and oleoresin were saturated fatty acids (SFAs), monounsaturated fatty acids (MUFAs), and polyunsaturated fatty acids (PUFAs). The SFAs (C8-C17) identified were caprylic acid (C8:0), lauric acid (C12:0), myristic acid (C14:0), palmitic acid (C16:0), and margaric acid (C17:0). Caprylic acid is a common eight-carbon fatty acid, which is abundantly found in coconut oil (Marina et al., 2009). Its systematic name is octanoic acid or caprylic acid. This fatty acid had a retention time of $19.14 \mathrm{~min}$. Its anion was detected at $m / z 143$. Lauric acid, also known as dodecanoic acid, retained as peak 36 of the negative ionization mode, which had $m / z 199$ (Figure 2). Similar to palmitic acid and myristic acid, both of these compounds detected at the chromatogram 

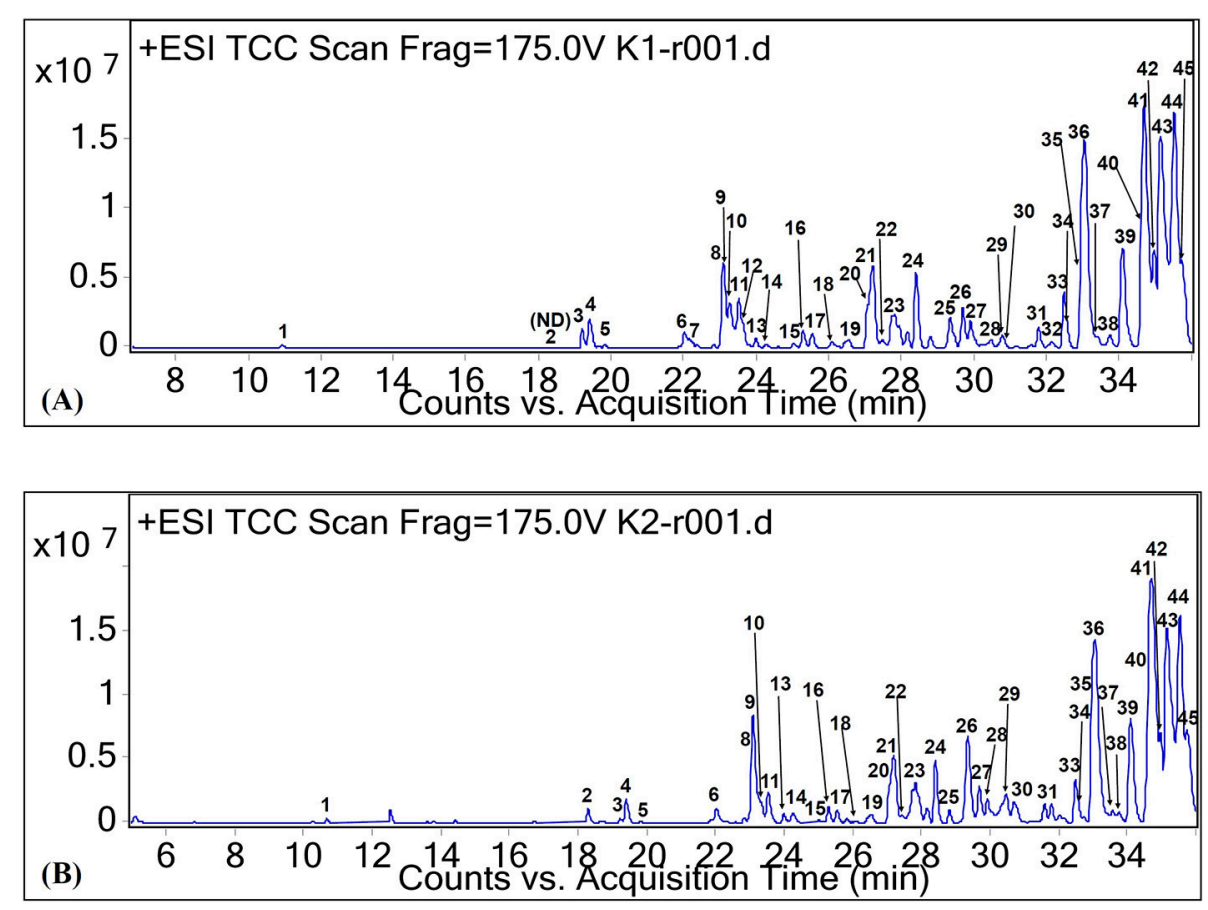

FIGURE 1 | Total ion chromatograms of CO oil (A) and oleoresin (B) based on positive ionization mode.

of negative ionization mode, had MS base peak at $\mathrm{m} / z 255$ and 227, respectively. However, palmitic acid was detected in the chromatogram of both ionization modes.

Among the SFAs identified in this study, margaric acid was detected at RT of $32.08 \mathrm{~min}$ (peak 32, Figure 1). It is also known as heptadecanoic acid. The cation of this fatty acid was tentatively identified as $m / z$ 293. It was only found in CO oil. All the other types of SFA were also tentatively identified in both CO oil and oleoresin. Although margaric acid seldom occurs in vegetable fat, it was found in CO oil at low concentration (based on the relative abundance value).

In this study, hexanoic acid (caproic acid, C6:0), decanoic acid (capric acid, C10:0), and octadecanoic acid (stearic acid, C18:0) were not detected in both CO oil and oleoresin. Lauric acid and myristic acid were the major fatty acids in $\mathrm{CO}$ oil and oleoresin, with relative abundance values higher than $2.45 \times 10^{6}$ unit. Lauric acid is also considered a medium-chain fatty acid.

On the other hand, the major MUFAs which were tentatively identified in both $\mathrm{CO}$ oil and oleoresin are palmitoleic acid $\left([\mathrm{M}+\mathrm{H}]^{+}\right.$at $\left.m / z 255\right)$ and oleic acid $\left([\mathrm{M}+\mathrm{H}]^{+}\right.$at $\left.m / z 283\right)$. As shown in Figure 1, both of these MUFAs were detected in the two fat samples with RT of $34.13 \mathrm{~min}$ (peak 39) and $35.46 \mathrm{~min}$ (peak 44). Palmitoleic acid (9-hexadecenoic acid) is an omega7 MUFA with molecular formula $\mathrm{C}_{16} \mathrm{H}_{30} \mathrm{O}_{2}$. It is an important fatty acid for the human body as it is made up of $5.6 \%$ of human epidermis (Carruthers, 1964). Besides the C16 MUFA, oleic acid (octadecenoic acid) was another MUFA found in the CO oil and oleoresin. Oleic acid in its cis-9 form was tentatively identified in the chromatograms of both positive and negative ionization modes, with the electron ionization MS of both cation and anion at $\mathrm{m} / z 283$ and 281, respectively. In fact, cis-9-oleic acid is the common fatty acid in plant oil (D'Evoli et al., 2015). Oleic acid is a C18 MUFA with various applications in the food industry and plays an important role in lipase synthesis. It has been reported to be able to induce lipase production during food processing, especially in the dairy industry (Aravindan et al., 2007).

In this study, we tentatively identified six PUFAs and other unclassified fatty acids. Among the naturally occurring PUFAs, linoleic acid (peak 31, Figure 1), $\alpha$-linolenic acid (peak 20, Figure 1), $\gamma$-linolenic acid (peak 19, Figure 1), di-homo- $\gamma$-linolenic acid (peak 38, Figure 1), and stearidonic acid (peak 16, Figure 1) were tentatively identified in both CO oil and oleoresin. For the comparison between $\alpha$-linolenic acid and $\gamma$-linolenic acid, a higher relative abundance value was observed for $\alpha$-linolenic acid than the value observed for $\gamma$-linolenic acid. Besides the relative abundance values, $\gamma$-linolenic acid eluted at $0.7 \mathrm{~min}$ faster than $\alpha$-linolenic acid. Based on the report by Momchilova and Nikolova-Damyanova (2003), the elution of fatty acid with more than one double bond in the longer carbon chain, which held itself stronger than the shorter chain. Therefore, a $\gamma$-linolenic acid which had the double bond with a shorter neighboring carbon chain (26.41 min) eluted faster than $\alpha$-linolenic acid with a longer carbon chain (27.11 min).

The other PUFAs identified were C18:2n6, C18:2n6, C18:2, $\mathrm{C} 18: 3 \mathrm{n} 3, \mathrm{C} 18: 3 \mathrm{n} 6, \mathrm{C} 20: 3 \mathrm{n} 6$, and C18:4n3. Linoleic acid was tentatively identified based on $[\mathrm{M}+\mathrm{H}]^{+}$and $[\mathrm{M}-\mathrm{H}]^{-}$at $m / z 281$ and $m / z$ 279. Both $\alpha$ - and $\gamma$-linolenic acid had $[\mathrm{M}+\mathrm{H}]^{+}$at $\mathrm{m} / z$ 279. This study shows that linolenic acid in its alpha form was the 

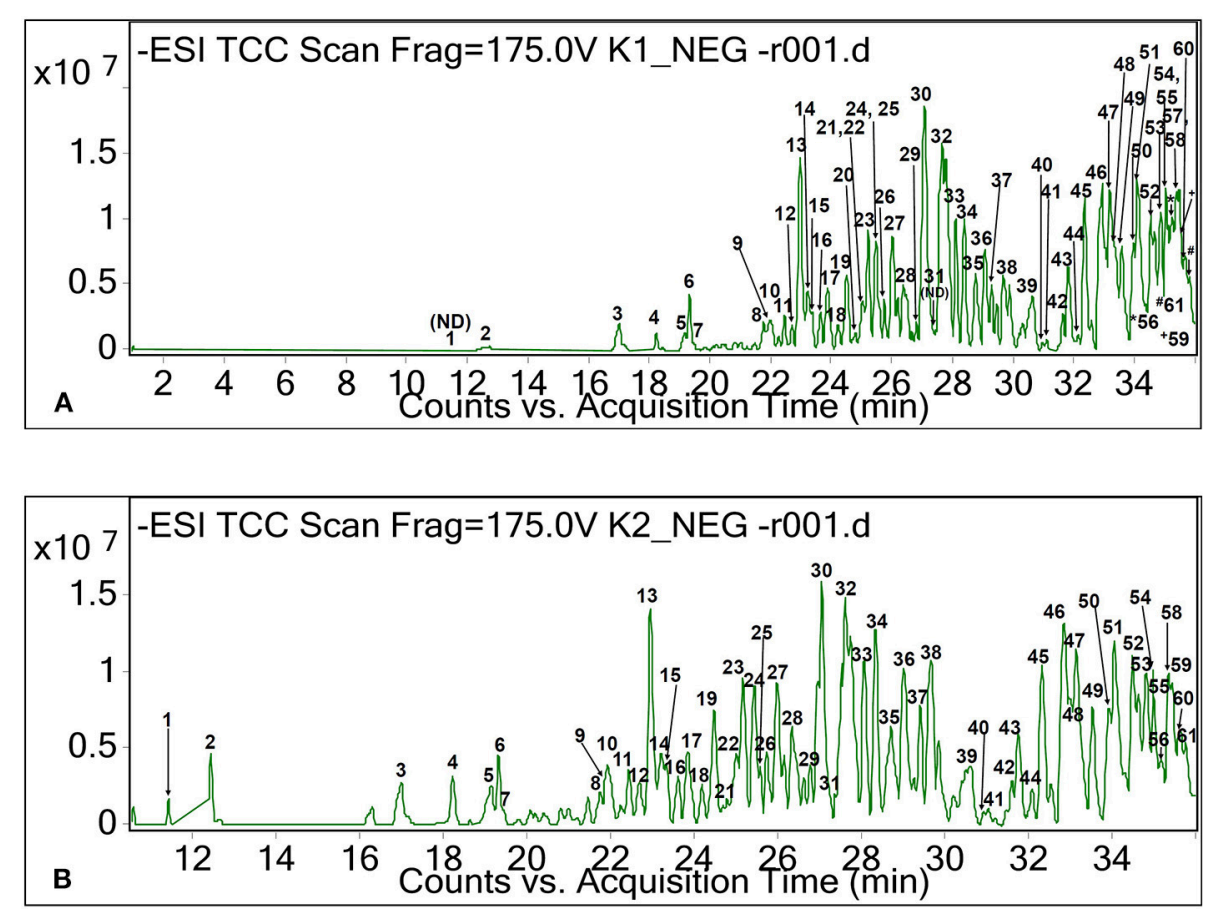

FIGURE 2 | Total ion chromatograms of CO oil (A) and oleoresin (B) based on negative ionization mode.

major compound with a high relative abundance value (Table 1), but the gamma form is one of the minor fatty acids in both $\mathrm{CO}$ oil and oleoresin. Di-homo- $\gamma$-linolenic acid $\left(\mathrm{C}_{20} \mathrm{H}_{34} \mathrm{O}_{2}\right)$ is the PUFA with longer carbon-chain; it was found to have $[\mathrm{M}+\mathrm{H}]^{+}$ at $m / z$ 307. Stearidonic acid is another omega-3 fatty acid found in both CO oil and oleoresin. It retained at $25.2 \pm 0.05 \mathrm{~min}$ of the chromatograms of both positive and negative ion modes. It had a monoisotopic mass of $276 \mathrm{Da}$.

Besides these main fatty acids, their isomers, hydroxylated, dihydroxylated, trihydroxylated, and epoxylated forms of fatty acids, as well as other chemical conformations, were tentatively identified for the CO oil and oleoresin. As shown in Table 1, these compounds had $[\mathrm{M}+\mathrm{H}]^{+}$and $[\mathrm{M}-\mathrm{H}]^{-}$at between $\mathrm{m} / z$ 157 and 421. Methyloctanoic acid $\left(\mathrm{C}_{9} \mathrm{H}_{18} \mathrm{O}_{2}\right.$, monoisotopic mass of $158 \mathrm{Da}$, peak 10 of Figure 2) was the methylated fatty acid with the least molecular weight, whereas (E,E)-3,7,11-trimethyl2,6,10-dodecatrienyl decanoic acid (peak 36, Figure 2) was a large compound, and it has capric acid as its base unit. This compound was only detected in CO oleoresin. Although capric acid (decanoic acid) was not detected in the fat samples, our finding shows that it existed in methylated forms. Due to the data being obtained from the anionic and cationic masses, we were not able to determine the position of all methylated fatty acids. Besides the anion of peak 36, (+)-3-O-myristoyl-L-1isovalerin (peak $35,[\mathrm{M}+\mathrm{H}]^{+}$at $m / z$ 387) was detected only in $\mathrm{CO}$ oleoresin. We concluded that only two lipids were specifically detected in $\mathrm{CO}$ oleoresin. In addition to these findings, the results show that the identified monoglycerides and diglycerides were part of the fat components in CO oil and oleoresin.
As shown in Figure 1 and Table 1, other lipids including phytosphingosine (peak 4, RT $19.42 \mathrm{~min},[\mathrm{M}+\mathrm{H}]^{+}$at $\mathrm{m} / z$ 318), dimethyl-safingol (peak 15, RT 24.98, $[\mathrm{M}+\mathrm{H}]^{+}$at $\mathrm{m} / z$ 330), and phospholipid (peak 44, RT $35.48 \mathrm{~min},[\mathrm{M}-22]^{+}$at $\mathrm{m} / z$ $553)$ were also tentatively identified in the oil and oleoresin. Due to the tentative identification of lipids being performed based on monoisotopic masses, we could not identify the type of phospholipid detected in the fat samples. Nevertheless, sphinganine (peak 6 of Figure 1, RT $22.13 \mathrm{~min},[\mathrm{M}+\mathrm{H}]^{+}$at $\mathrm{m} / z$ 302) was detected in CO oil. Based on the relative abundance values of the MS spectra, these lipids were the minor compounds detected in the CO oil and oleoresin.

In this study, lauric acid, myristic acid, $\alpha$-linolenic acid, dihydroxystearic acid, and epoxy-hydroxystearic acid were relatively abundant in the fat samples. These compounds can be considered as the major fatty acids in CO oil and oleoresin due to their high relative abundance values. Based on these findings, we could conclude that $\mathrm{CO}$ oil and oleoresin have a balanced fatty acid composition, with almost equal levels of SFA, MUFA, and PUFA. The relative abundance values also proved that major fatty acids were tentatively identified as methyloctanoic acid (a methylated form of caprylic acid), palmitic acid, oleic acid, oxo-oleic acid, trihydroxyoctadecenoic acid, hydroperoxyoctadecadienoic acid, hydroxy-oxo-octadecadienoic acid, octadecenedioic acid, stearidonic acid, octadecadiynoic acid, and heneicosanedioic acid. The levels of these long-chain fatty acids especially epoxyhydroxystearic acid and dihydroxystearic acid were found to be in a higher amount in $\mathrm{CO}$ oleoresin than in $\mathrm{CO}$ oil. The result 
shows that the level of dihydroxystearic acid was 2.35 times higher in $\mathrm{CO}$ oleoresin than in the oil. These long-chain fatty acids might contribute to the high viscosity of $\mathrm{CO}$ oleoresin compared to that of the $\mathrm{CO}$ oil.

Monoolein $\left(\mathrm{C}_{21} \mathrm{H}_{40} \mathrm{O}_{4},[\mathrm{M}-22]^{+}\right.$at $m / z$ 357, peak 42 of Figure 1), monopalmitin $\left(\mathrm{C}_{19} \mathrm{H}_{38} \mathrm{O}_{4},[\mathrm{M}+\mathrm{H}]^{+}\right.$at $m / z 331$, peak 43 of Figure 1), and monolinolein $\left(\mathrm{C}_{21} \mathrm{H}_{38} \mathrm{O}_{4},[\mathrm{M}+\mathrm{H}]^{+}\right.$at $\mathrm{m} / z$ 355, peaks 33 and 36 of Figure 1), as well as diglyceride $\left(\mathrm{C}_{40} \mathrm{H}_{64} \mathrm{O}_{5},[\mathrm{M}-2 \mathrm{H}]^{-} 2\right.$ at $\mathrm{m} / z$ 311, peak 15 of Figure 2$)$ were detected as the major fat components in the fat samples. The result also showed that 1-monolinolein (peak 36 of Figure 1) was the only major glyceride in the fat samples. Monoolein was also found to be relatively abundant in the fat samples. In addition to these fatty acids, lanosterol and its hydroxyl forms were tentatively identified in both of the $\mathrm{CO}$ fat samples. As shown in Table 1, at RT $35.7 \mathrm{~min}$, lanosterol $\left(\mathrm{C}_{30} \mathrm{H}_{48} \mathrm{O}\right.$, peak 46 of Figure 1) and its hydroxyl-forms (peaks 45 and 46) were the major plant sterols in the CO fruit.

Five diglycerides were detected in both fat samples (Table 1). Although these diglycerides could have several chemical conformations, lipid with the masses found may only correspond to the structures of diglycerides as suggested in the LIPID MAPS database. According to the database from LIPID MAPS, a lipid with monoisotopic mass of $624 \mathrm{Da}$ and the formula of $\mathrm{C}_{40} \mathrm{H}_{64} \mathrm{O}_{5}$ may correspond to DG(17:2(9Z,12Z)/20:5(5Z,8Z,11Z,14Z,17Z) or DG(15:1(9Z)/22:6(4Z,7Z,10Z,13Z,16Z,19Z). The LIPID MAPS database also suggested that anions of peaks 45 and 49 could be triglycerides. However, only one of these diglycerides was found in the $\mathrm{CO}$ oil, which had the molecular formula of $\mathrm{C}_{58} \mathrm{H}_{92} \mathrm{O}_{6}\left([\mathrm{M}-\mathrm{H}]^{-}\right.$at $m / z 884.7$, peak 57 of Figure 2$)$. The molecular structure of this glyceride was unknown.

A wide range of fatty acids was determined in the oil and oleoresin of the CO fruit. Some of these fatty acids have either positive or negative effects on human health. $\mathrm{CO}$ oil also has a balanced amount of both saturated and unsaturated fatty acids. Saturated fatty acids are known to have detrimental effects, especially in terms of increased risk of cardiovascular diseases and stroke (Siri-Tarino et al., 2010). Among the different lengths of saturated fatty acids, medium-chain triglycerides have protective effects against cardiovascular diseases and many other diseases.

Lauric acid is one of the medium-chain triglycerides that was detected in the $\mathrm{CO}$ fat samples in a relatively high amount. Previous studies showed that a diet rich in lauric acid is less likely to promote obesity. Lauric acid was also found to be able to improve the lipid profile from the experimental trials (McCarty and DiNicolantonio, 2016). As compared to lauric acid (C12), myristic acid (C14) is considered as the long-chain fatty acid. Although myristic acid has only two carbon chains longer than those of lauric acid, it poses deleterious effects on human health. Same with palmitic acid, a diet rich in myristic acid could increase the risk of cardiovascular diseases following the rise of LDL-cholesterol and apolipoprotein B levels in healthy men and women (Zock et al., 1994).

Previous studies reported that monoglycerides and diglycerides had protective effects against several chronic diseases (Feltes et al., 2013). Due to the anti-diabetic and antiatherogenic effects of monoolein (Cho et al., 2010), the high level of monoglyceride in $\mathrm{CO}$ oil and oleoresin makes these fat samples as potential functional foods. Diglyceride is a prospective therapeutic agent for antiobesity. The effect is probably due to the increased $\beta$-oxidation of the fatty acids found during the metabolism of the diglyceride (Lo et al., 2008).

In addition to the balance fatty acid composition, the $\mathrm{CO}$ oil has a low level of linoleic acid. The level of linoleic acid in human blood is the key to the development of several chronic diseases (Spiteller, 1998). Therefore, a balanced fatty acid composition of the vegetable oil is essential for maintaining good health besides providing oxidative stability.

In this study, we found a desirable level of isanic acid in $\mathrm{CO}$ oil and oleoresin. Isanic acid, also known as erythrogenic acid $\left(\mathrm{C}_{18} \mathrm{H}_{26} \mathrm{O}_{2}\right)$, was tentatively identified as peak 18 (Figure 1). It had protonated molecular ion $[\mathrm{M}+\mathrm{H}]^{+}$at $\mathrm{m} / z 275$, and it was detected only in $\mathrm{CO}$ oil. However, 8-hydroxyisanic acid $\left(\mathrm{C}_{18} \mathrm{H}_{28} \mathrm{O}_{3}\right)$ was found in both of the $\mathrm{CO}$ fat samples, with the RT of $26.07 \mathrm{~min}$ (Table 1). Isanic acid is also a less common fatty acid found in vegetable oil. It had been first detected in Ongokea gore seed (isano) oil from African countries (Ntumba et al., 2015). It is known as a non-edible fatty acid. The literature showed that isanic acid turns to a vivid red when exposed to light at increasing temperature (Black and Weedon, 1953). It also contributes to the color of the oil besides carotenoids. Although isanic acid was detected in the CO oil, our previous study proved that the oil is not hepatotoxic (Faridah Hanim et al., 2015).

Based on the FAMEs analysis, the main component of $\mathrm{CO}$ oil was palmitic acid (41.53 $\pm 0.13 \%)$, followed by oleic acid (38.79 $\pm 0.01 \%)$, and (cis) linoleic acid (11.95 $\pm 0.01 \%)$. The fatty acid composition of the $\mathrm{CO}$ oil was quantified and tabulated in Table 2. CO oil was characterized as SFA-rich oil due to its high SFA composition (47.62 $\pm 0.13 \%)$. However, the MUFA and PUFA in CO oil cannot be disregarded. CO oil contained 39.93 $\pm 0.16 \%$ MUFA and $12.48 \pm 0.01 \%$ PUFA. Among the SFA, no short-chain fatty acids were detected in the oil.

The saturated carbon chains detected were C8, C11, and C12. These carbon chains were determined as caprylic acid, undecanoic acid, and lauric acid, as well as long carbon chains $\mathrm{C} 14-\mathrm{C} 24$. Based on the carbon chains, these fatty acids should be known as long-chain fatty acids. The fatty acids were named as follows: myristic acid (C14), pentadecanoic acid (C15), palmitic acid (C16), heptadecanoic acid (C17), stearic acid (C18), arachidic acid (C20), heneicosanoic acid (C21), behenic acid (C22), tricosanoic acid (C23), and lignoceric acid (C24).

Based on the results obtained, the SFA composition in the $\mathrm{CO}$ oil was mainly long-chain fatty acids. Although some of the carbon chains such as C15, C17, C21, and C23 are rarely found in fruit oils, these fatty acids were detected based on the carbon chains. Therefore, LCMS analysis was performed to confirm the existence of these fatty acids. The LCMS data showed that the fatty acids with carbon chains of C20-C24 were not detected in both $\mathrm{CO}$ oil and oleoresin. These carbon chains could be derived from the other types of lipid or hydrocarbon-containing compounds. 
TABLE 2 | Fatty acid composition of Canarium odontophyllum oil extracted with supercritical carbon dioxide.

\begin{tabular}{|c|c|c|c|}
\hline Carbon & FAMEs & $\%$ in fat & $\mathrm{mg} / 100 \mathrm{~g}$ \\
\hline $\mathrm{C} 8$ & Caprylic & $0.05 \pm 0.00$ & $47.94 \pm 2.00$ \\
\hline C10 & Capric & $0.01 \pm 0.00$ & $5.99 \pm 0.14$ \\
\hline C11 & Undecanoic & $0.01 \pm 0.01$ & $9.50 \pm 6.76$ \\
\hline C12 & Lauric & $0.72 \pm 0.07$ & $694.46 \pm 38.38$ \\
\hline C13 & Tridecanoic & $0.00 \pm 0.00$ & $3.81 \pm 0.66$ \\
\hline C14 & Myristic & $0.28 \pm 0.00$ & $270.41 \pm 10.39$ \\
\hline C15 & Pentadecanoic & $0.03 \pm 0.00$ & $25.42 \pm 1.27$ \\
\hline C16 & Palmitic & $41.53 \pm 0.13$ & $40273.79 \pm 1517.22$ \\
\hline C17 & Heptadecanoic & $0.11 \pm 0.00$ & $108.37 \pm 5.49$ \\
\hline C18 & Stearic & $4.31 \pm 0.01$ & $4175.36 \pm 167.51$ \\
\hline $\mathrm{C} 20$ & Arachidic & $0.10 \pm 0.00$ & $93.08 \pm 4.36$ \\
\hline $\mathrm{C} 21$ & Henicosanoic & $0.02 \pm 0.01$ & $18.51 \pm 11.23$ \\
\hline $\mathrm{C} 22$ & Behenic & $0.25 \pm 0.07$ & $245.22 \pm 77.32$ \\
\hline $\mathrm{C} 23$ & Tricosanoic & $0.11 \pm 0.00$ & $106.69 \pm 1.89$ \\
\hline \multirow[t]{2}{*}{$\mathrm{C} 24$} & Lignoceric & $0.10 \pm 0.01$ & $97.12 \pm 16.33$ \\
\hline & Total SFA & $47.62 \pm 0.13$ & $46175.65 \pm 1746.62$ \\
\hline C14:1 & Myristoleic & $0.04 \pm 0.00$ & $40.51 \pm 0.3$ \\
\hline C15:1 & Cis-10-pentadecenoic & $0.04 \pm 0.00$ & $34.59 \pm 0.97$ \\
\hline C16:1 & Palmitoleic & $0.64 \pm 0.01$ & $615.88 \pm 24.31$ \\
\hline C17:1 & Cis-10-heptadecanoic & $0.03 \pm 0.00$ & $25.20 \pm 0.97$ \\
\hline C18:1n9c & Oleic & $38.79 \pm 0.01$ & $37616.98 \pm 1541.0$ \\
\hline C20:1n9 & Cis-11-eicosenoic & $0.07 \pm 0.01$ & $29.41 \pm 41.58$ \\
\hline C22:1n9 & Erucic & $0.04 \pm 0.03$ & $39.27 \pm 27.63$ \\
\hline \multirow[t]{2}{*}{$\mathrm{C} 24: 1$} & Nervonic & $0.30 \pm 0.13$ & $289.66 \pm 146.71$ \\
\hline & Total MUFA & $39.93 \pm 0.16$ & $38691.48 \pm 1783.48$ \\
\hline C18:2n6c & Linoleic (cis) & $11.95 \pm 0.01$ & $11593.46 \pm 456.69$ \\
\hline C18:3n6 & $\gamma$-linolenic & $0.11 \pm 0.00$ & $103.92 \pm 1.88$ \\
\hline C18:3n3 & $\alpha$-linolenic & $0.40 \pm 0.00$ & $387.11 \pm 15.2$ \\
\hline C20:4n6 & Arachidonic & $0.02 \pm 0.01$ & $14.71 \pm 4.12$ \\
\hline Total PUFA & & $12.48 \pm 0.01$ & $12099.20 \pm 477.88$ \\
\hline
\end{tabular}

\section{Phenolics and Terpenoids}

The phenolic compounds identified in most of the fat and oil were in semi-polar to non-polar forms. In this study, we managed to tentatively identify semi-polar coumaric acid and anisic acid as the main phenolic acids in CO oil and oleoresin. Although the relative abundance values of these phenolic acids were not as high as those observed in the major fatty acids, a low amount of these compounds has a good effect on human health because phenolics are strong antioxidants. Peak 3 was tentatively identified as the peak consisting of a mixture of flavonoids at RT of $19.36 \mathrm{~min}$ (Table 3; Figure 1), including prenyl-naringenin, sophoraflavanone $\mathrm{B}$, and neobavaisolavone. The anions of these compounds had electron ionization mass spectra at $\mathrm{m} / \mathrm{z} 340,340$, and 322 respectively. Peak 3 was also tentatively identified as either matairesinol or pinoresinol. These phenolics were the minor compounds tentatively identified in both of the fat samples.

As shown in Table 3, triterpenes, sesquiterpenes, and apocarotenoids were tentatively identified in the $\mathrm{CO}$ oil and oleoresin. Based on the relative abundance value of the peak, a moderate level of hydroxy-apocarotenal $\left(\mathrm{C}_{27} \mathrm{H}_{36} \mathrm{O}_{2}\right)$ was retained at 35.3-35.4 min (Figure 2) with $[\mathrm{M}-\mathrm{H}]^{-}$at $\mathrm{m} / \mathrm{z} 391$ (Table 3). It is one of the carotenoids found in fruits (Gross and Eckhardt, 1981). Peaks 58 and 59 of the chromatogram of negative ionization mode (Figure 2) for both $\mathrm{CO}$ oil and oleoresin were tentatively identified as two isomers of hydroxyapocarotenal. On the other hand, cincassiol $\mathrm{B}\left(\mathrm{C}_{20} \mathrm{H}_{32} \mathrm{O}_{8}\right)$ was only determined in $\mathrm{CO}$ oil. It gave a protonated molecular ion $[\mathrm{M}+\mathrm{H}]^{+}$at $m / z 401$ (peak 7, Figure 1). Peak 1 was tentatively identified only in the $\mathrm{CO}$ oleoresin as lacinilene $\mathrm{C}\left(\mathrm{C}_{15} \mathrm{H}_{18} \mathrm{O}_{3}\right)$ with RT $13.76 \mathrm{~min}$ and $[\mathrm{M}+\mathrm{H}]^{+}$at $\mathrm{m} / z$ 247. Also, phaseollin and kauralexin A2 together with oleanolic acid and oleanonic aldehyde were the terpenoids identified in both fat samples (Table 3).

The yellow hue of $\mathrm{CO}$ oil could be contributed by both apocarotenal and isanic acid. A previous study reported that apocarotenal has provitamin A activity (Bagdon et al., 1962). It is considered an intermediary of the conversion of $\beta$-carotene to vitamin A. Although the provitamin A activity is not as high as $\beta$-carotene, consumption of $\mathrm{CO}$ may improve the plasma vitamin A status of a healthy individual. Prasad et al. (2011) also reported that a moderate amount of $\beta$-carotene was determined in the saponified extract of $\mathrm{CO}$ fruit. In addition to the apocarotenal, carotenoids were not identified in the $\mathrm{CO}$ fat due to several reasons, one of which is that carotenoids occur in the plant as carotenoid-protein complexes and carotenoid glycosides (Jürgens and Weckesser, 1985).

\section{Volatiles and Other Aromatic Compounds}

Table 4 shows the volatiles and other aromatic compounds identified in the CO oil and oleoresin. The use of LCMS in detecting volatiles causes a loss of most known volatiles in the fat samples. The commonly known volatiles identified in both $\mathrm{CO}$ oil and oleoresin were cuparene $\left([\mathrm{M}+\mathrm{H}]^{+}\right.$at $\mathrm{m} / z$ 202) and hexadecenal $\left([\mathrm{M}+\mathrm{H}]^{+}\right.$at $\mathrm{m} / z$ 238). In this study, 4-(2-hydroxy-propoxy)-3,5-dimethylphenol $\left([\mathrm{M}+\mathrm{H}]^{+}\right.$ at $\mathrm{m} / z$ 196) and 1,2-dioctanoyl-1,2,3-butanetriol $\left([\mathrm{M}+\mathrm{H}]^{+}\right.$at $\mathrm{m} / z$ 358) were tentatively identified as the novel volatiles in $\mathrm{CO}$ oil. The volatiles were not detected in $\mathrm{CO}$ oleoresin. In addition to volatiles, other aromatic compounds $\left([\mathrm{M}+\mathrm{H}]^{+}\right)$ identified in the fat samples were deoxyshikonin $(\mathrm{m} / \mathrm{z}$ 273, peak 10), 4-prenylphlorisovalerophenone $(\mathrm{m} / z$ 279, peak 25), demethylphylloquinol $(m / z \quad 439$, peak 33), and demethylphylloquinone $(\mathrm{m} / \mathrm{z} 437$, peak 34$)$, found based on positive ionization mode (Table 4). The other quinol-based compounds identified were also not found in most of the commercial fruits.

Literature shows that more than 30 volatiles were tentatively identified in the fruit of Canarium pimela (Lv et al., 2014). The fruit is from the genus Canarium, which should have a similar characteristic as found in the $\mathrm{CO}$ fruit. However, the volatiles found in the samples were far lesser than the volatiles reported in the previous study. It could be due to the loss of most of the volatiles in the sample during the $\mathrm{SCO}_{2}$ extraction of the $\mathrm{CO}$ oil. Therefore, only cuparene and hexadecenal were the major volatiles retained in the fat samples. This observation is supported by the finding from a previous study, where supercritical fluid extracted essential oils had lower percentages 


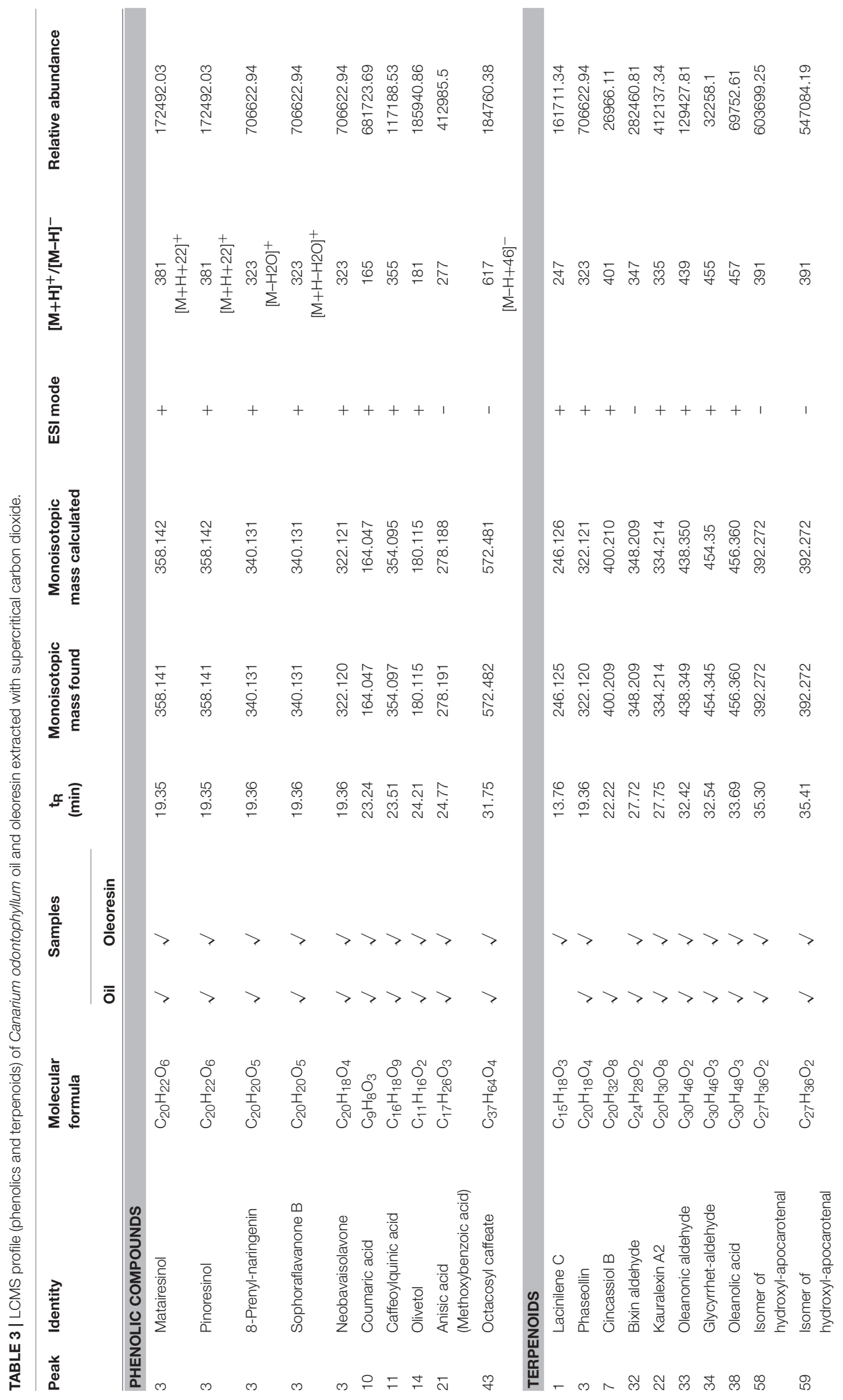




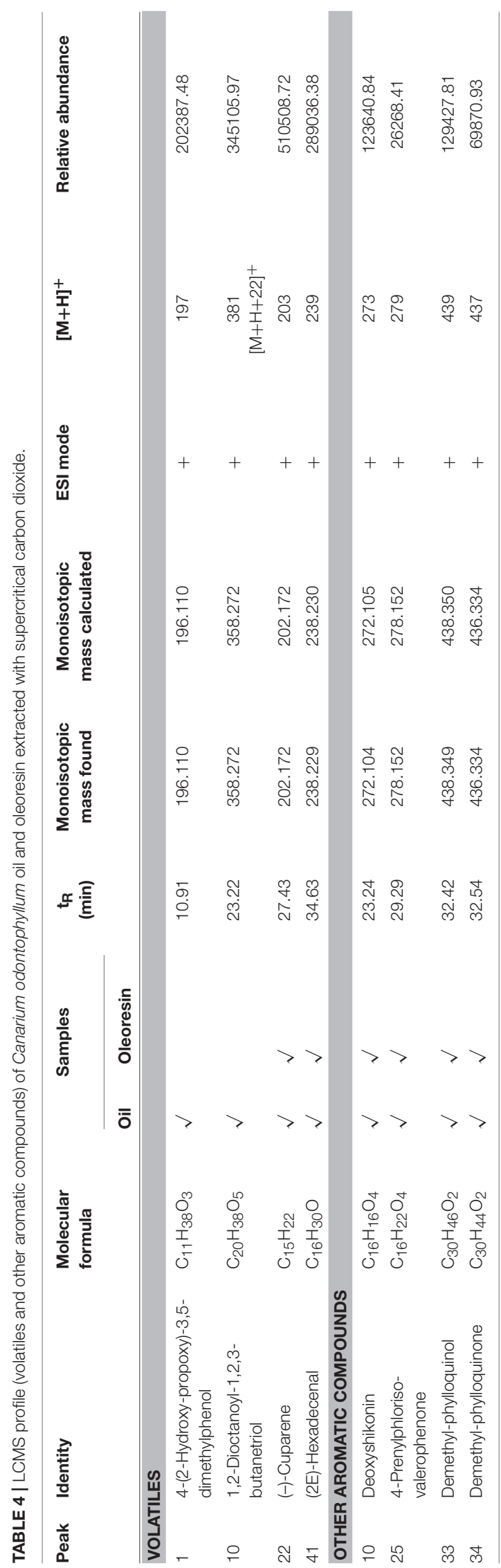

of volatiles than the essential oils extracted using simultaneous distillation-extraction apparatus (Díaz-Maroto et al., 2002).

Deoxyshikonin is one of the naphthoquinones found in plant. It has a molecular mass of $272 \mathrm{Da}$ with a formula structure of $\mathrm{C}_{16} \mathrm{H}_{16} \mathrm{O}_{4}$. It has never been reported in fruit, except roots and barks. Therefore, deoxyshikonin is the first to be identified in the fruit of Canarium. According to Song (2015), valerophenone is the metabolite produced during strawberry ripening. Besides valerophenone, demethylphylloquinone is a fat-soluble compound identified in the CO fat. It is another form of a fat-soluble vitamin, also known as vitamin $\mathrm{K}$, which is present naturally in most plants. The occurrence of these quinolbased compounds may add value to the $\mathrm{CO}$ oil and oleoresin.

\section{Peptides, Other Phytochemicals, and Unknown}

In this study, we tentatively identified a few peptide fragments in the $\mathrm{CO}$ oil and oleoresin. These peptide fragments had monoisotopic masses of 340.2-414.2 Da (Table 5). Trp-Ala-Pro, His-Met-Lys, and Phe-Asn-Ser were tentatively identified in both of the $\mathrm{CO}$ fat samples. These peptides had molecular ions $[\mathrm{M}+\mathrm{H}]^{+}$at $m / z 373,[\mathrm{M}+\mathrm{H}]^{+}$at $m / z 415$, and $[\mathrm{M}-\mathrm{H}]^{-}$at $m / z$ 365 respectively. However, Lys-Lys-Lys $\left([\mathrm{M}+\mathrm{H}]^{+}\right.$at $\mathrm{m} / z$ 403) and Pro-Lys-Pro $\left([\mathrm{M}-\mathrm{H}]^{-}\right.$at $m / z$ 339) were only identified in $\mathrm{CO}$ oil and oleoresin, respectively. Peptides with the molecular weights of higher than 1,000 Da were not able to be identified in the oil samples. It is due to our mass searching technique which was based only on the PlantCyc database and some major online chemical databases. Nevertheless, we had no access to the database for peptide sequences. Therefore, the peptides with more than three amino acids and polypeptides remained unknown. This study makes way for other researchers to work on the peptide sequencing for $\mathrm{CO}$ oil.

In nature, peptides are short-chain-linked amino acids with specific functions. The shortest peptide consists of two amino acids, whereas the peptide chain can be as long as a chain of 50 amino acids. In fact, peptide sequencing is typically performed using MALDI-MS. In this study, the three amino acid peptides were tentatively identified. Although these identified peptides do not have known functional properties, peptides have been reported to possess protective effect against in vitro oxidative stress (Ioudina and Uemura, 2003).

Besides peptides, eight other known compounds were tentatively identified in the fat samples (Table 5). The results showed that hercynine $\left([\mathrm{M}+\mathrm{H}]^{+}\right.$at $\mathrm{m} / z$ 198) and $10-(\beta-$ dimethyl-aminopropionyl)phenothiazine $\left([\mathrm{M}-\mathrm{H}]^{-}\right.$at $\mathrm{m} / z$ 298) were only found in the $\mathrm{CO}$ oleoresin. These compounds are the novel compounds identified in the fruit oil. Hercynine is the intermediate compound of ergothioneine biosynthesis in fungus (Askari and Melville, 1962). The other six organic compounds detected in the $\mathrm{CO}$ fat had $[\mathrm{M}-\mathrm{H}]^{-}$ranged between $\mathrm{m} / \mathrm{z} 226$ and 493 (Table 5), which include the derivatives of two heterocyclic amines. One of these six compounds, dodecyl hydroxyphosphinate (peak 39 of Figure 2), was a novel fatty acid derivative that was abundantly found in the fat samples. It was also one of the major compounds in the CO fat samples. 


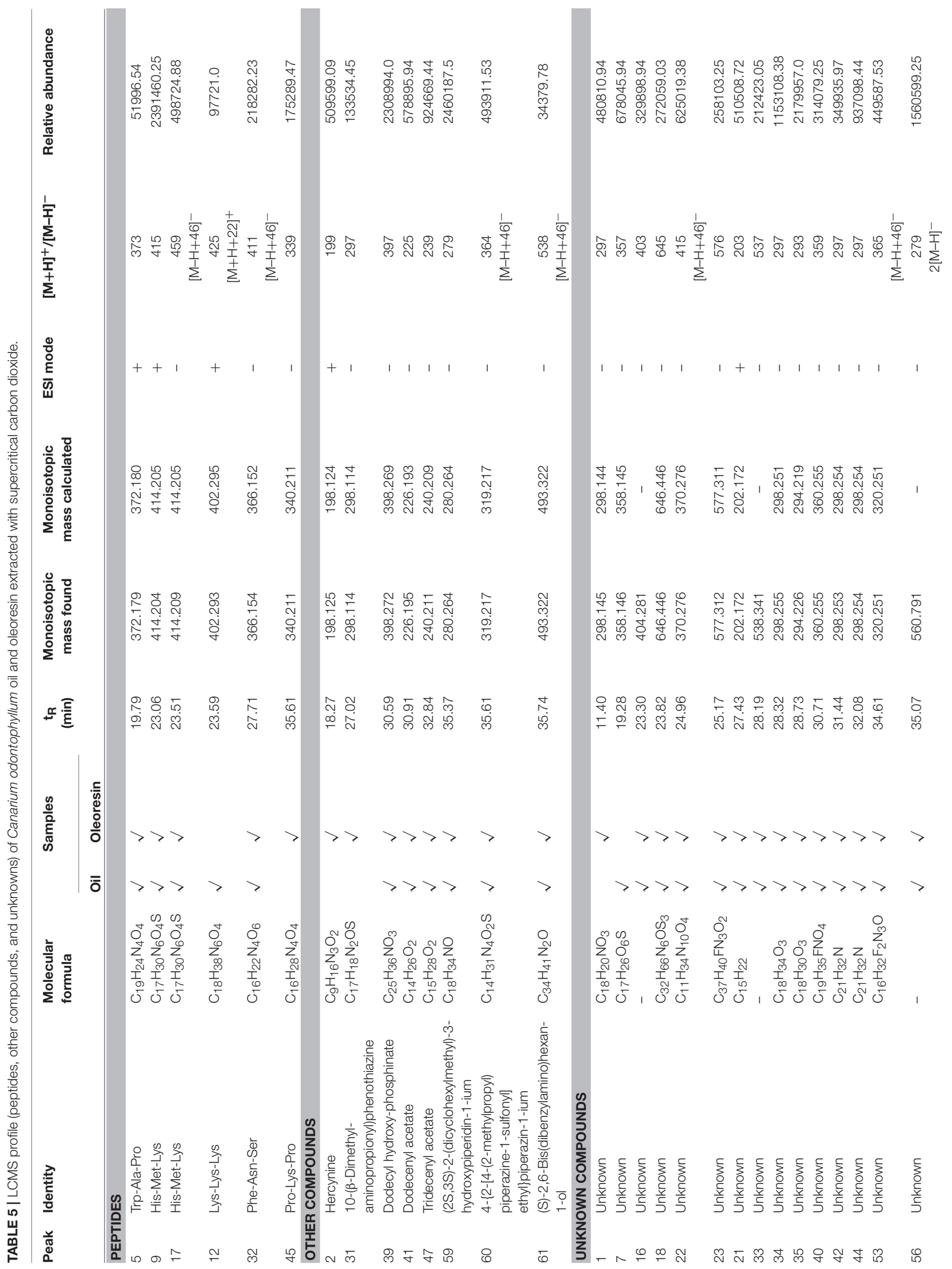


The detection of this compound in the $\mathrm{CO}$ oil could be due to fungal contamination on the $\mathrm{CO}$ peel. On top of fungal contamination, 10-( $\beta$-Dimethyl-aminopropionyl) phenothiazine, which is possibly derived from pesticide, was detected in the $\mathrm{CO}$ oleoresin. During the searching and matching of monoisotopic masses of the compounds with the available chemical databases, we found many unmatched compounds. Therefore, these unmatched compounds were named as unknowns. These unknowns had monoisotopic masses ranged between 202.17 and $646.45 \mathrm{Da}$.

Two unknown compounds were found to be the major compounds in the fat samples-they were relatively abundant in both $\mathrm{CO}$ oil and oleoresin. One of the compounds had a molecular formula of $\mathrm{C}_{18} \mathrm{H}_{34} \mathrm{O}_{3}$, while the molecular formula of another unknown compound was determined. As shown in Table 5, these unknown compounds retained at RT $28.3 \mathrm{~min}$ and $35.1 \mathrm{~min}$, respectively. Also, some were the fragment ions of an unknown compound. As this is the first study conducted to identify potential chemical substances and phytochemicals in the $\mathrm{CO}$ oil and oleoresin, we merely reported the compounds identified in the $\mathrm{CO}$ oil and oleoresin based on the monoisotopic masses obtained from the LC-ESI-MS analysis.

\section{CONCLUSION}

The LC-ESI-MS analysis gave a full insight into the potent functional compounds and nutritional components in the $\mathrm{SCO}_{2}$ extracted $\mathrm{CO}$ oil and oleoresin. $\mathrm{CO}_{2}$ is the ideal type of organic solvent for the extraction of fat-soluble compounds from plant materials. The $\mathrm{CO}_{2}$ extracted $\mathrm{CO}$ fat samples had lauric acid, myristic acid, and $\alpha$-linolenic acid as the major fatty acids in both $\mathrm{CO}$ oil and oleoresin. 1-Monolinolein was

\section{REFERENCES}

Aravindan, R., Anbumathi, P., and Viruthagiri, T. (2007). Lipase applications in food industry. Ind. J. Biotechnol. 6, 141-158.

Askari, A., and Melville, D. B. (1962). The reaction sequence in ergothioneine biosynthesis: hercynine as an intermediate. J. Biol. Chem. 237, 1615-1618.

Azlan, A., Prasad, K. N., Khoo, H. E., Abdul-Aziz, N., Mohamad, A., Ismail, A., et al. (2010). Comparison of fatty acids, vitamin E and physicochemical properties of Canarium odontophyllum Miq. (dabai), olive and palm oils. J. Food Compos. Anal. 23, 772-776. doi: 10.1016/j.jfca.2010.03.026

Bagdon, R. E., Impellizzeri, C., and Osadca, M. (1962). Studies on the toxicity and metabolism of $\beta$-apo- $8^{\prime}$-carotenal in dogs. Toxicol. Appl. Pharmacol. 4, 444-456. doi: 10.1016/0041-008X(62)90031-5

Basri, D. F., Rahman, N. S. A., Ali, S. S., and Zainalabidin, S. (2018). The vasorelaxant effect of Canarium odontophyllum Miq. (dabai) extract in rat thoracic aorta. J. Basic Appl. Sci. 5, 75-79. doi: 10.1016/j.ejbas.2017.11.004

Biswas, B., Rogers, K., McLaughlin, F., Daniels, D., and Yadav, A. (2013). Antimicrobial activities of leaf extracts of guava (Psidium guajava L.) on two Gram-negative and Gram-positive bacteria. Int. J. Microbiol. 2013:746165. doi: $10.1155 / 2013 / 746165$

Black, H. K., and Weedon, B. C. L. (1953). Unsaturated fatty acids. Part I. The synthesis of erythrogenic (isanic) and other acetylenic acids. J. Chem. Soc. 40, 1785-1793. doi: 10.1039/jr9530001785

Carruthers, C. (1964). Fatty acid composition of the phosphatide and triglyceride fractions of human epidermis. Proc. Soc. Exp. Biol. Med. 115, 215-218. doi: 10.3181/00379727-115-28873 also the major glyceride in the fat samples; it contains a single fatty acid chain that was covalently bonded through an ester linkage to a glycerol molecule. Two unknown compounds and a peptide (His-Met-Lys) were tentatively identified as the main components of the fat samples. Besides these compounds, phenolics, terpenoids, volatiles, and other aromatic compounds were tentatively identified in both $\mathrm{CO}$ oil and oleoresin.

Based on these findings, (E,E)-3,7,11-trimethyl-2,6,10dodecatrienyl decanoic acid, (+)-3-O-myristoyl-L-1-isovalerin, lacinilene $\mathrm{C}$, hercynine, and 10-( $\beta$-dimethyl-aminopropionyl) phenothiazine were the lipids found only in the CO oleoresin. This study has its limitation, where the compounds were only tentatively identified using the LCMS technique. Based on the relative abundance value, a higher level of the long-chain fatty acid such as dihydroxystearic acid was observed in the $\mathrm{CO}$ oleoresin than in the oil. Although many compounds are considered as unknown, they have not been identified in the CO fat samples. Future studies may focus on the characterization and identification of these two major unknowns in the $\mathrm{CO}$ fat using other analytical technique or a more advanced technology.

\section{AUTHOR CONTRIBUTIONS}

$\mathrm{HK}$ and AA designed this experiment. HK and NA performed the experiments and analyzed the data. HK wrote the manuscript. $\mathrm{AA}$ and NA revised and proofread the manuscript.

\section{FUNDING}

This study was supported financially by the Innovation Development Research Grant from Universiti Putra Malaysia (Vote Number 9449700).

Chew, L. Y., Prasad, K. N., Amin, I., Azrina, A., and Lau, C. Y. (2011). Nutritional composition and antioxidant properties of Canarium odontophyllum Miq. (dabai) fruits. J. Food Compos. Anal. 24, 670-677. doi: 10.1016/j.jfca.2011.01.006

Cho, K. H., Hong, J. H., and Lee, K. (2010). Monoacylglycerol (MAG)-oleic acid has stronger antioxidant, anti-atherosclerotic, and protein glycation inhibitory activities than MAG-palmitic acid. J. Med. Food 13, 99-107. doi: 10.1089/jmf.2009.1024

D’Evoli, L., Lucarini, M., Gabrielli, P., Aguzzi, A., and Lombardi-Boccia, G. (2015). Nutritional value of Italian pistachios from Bronte (Pistacia vera, L.), their nutrients, bioactive compounds and antioxidant activity. Food Nutr. Sci. 6, $1267-1276$

Díaz-Maroto, M. C., Perez-Coello, M. S., and Cabezudo, M. D. (2002). Supercritical carbon dioxide extraction of volatiles from spices: comparison with simultaneous distillation-extraction. J. Chromatogr. A 947, 23-29. doi: 10.1016/S0021-9673(01)01585-0

Faridah Hanim, S., Azrina, A., Khoo, H. E., and Amin, I. (2015). Protective effects of pulp and kernel oils from Canarium odontophyllum fruit in normal and hypercholesterolemic rabbits. Int. Food Res. J. 22, 1318-1326.

Feltes, M. M. C., de Oliveira, D., Block, J. M., and Ninow, J. L. (2013). The production, benefits, and applications of monoacylglycerols and diacylglycerols of nutritional interest. Food Bioprocess Tech. 6, 17-35. doi: 10.1007/s11947-012-0836-3

Gross, J., and Eckhardt, G. (1981). Structures of persicaxanthin, persicachrome and other apocarotenols of various fruits. Phytochemistry 20, 2267-2269. doi: 10.1016/0031-9422(81)80127-6 
Güçlü-Üstündag, Ö., and Temelli, F. (2004). Correlating the solubility behavior of minor lipid components in supercritical carbon dioxide. J. Supercrit. Fluids 31, 235-253. doi: 10.1016/j.supflu.2003.12.007

Ioudina, M., and Uemura, E. (2003). A three amino acid peptide, Gly-Pro-Arg, protects and rescues cell death induced by amyloid $\beta$-peptide. Exp. Neurol. 184, 923-929. doi: 10.1016/S0014-4886(03)00314-5

IUPAC (1987). Method 2.301: Standard Methods for the Analysis of Oils, Fats and Derivatives, 7th Edition. Durham, NC: International Union of Pure and Applied Chemistry.

Jelani, N. A. A., Azlan, A., Ismail, A., Khoo, H. E., and Alinafiah, S. M. (2017). Fatty acid profiles and antioxidant properties of dabai oil. ScienceAsia 43, 347-353. doi: 10.2306/scienceasia1513-1874.2017.43.347

Jürgens, U. J., and Weckesser, J. (1985). Carotenoid-containing outer membrane of Synechocystis sp. strain PCC6714. J. Bacteriol. 164, 384-389.

Khoo, H. E. (2014). Phenolic Content, Antioxidative Properties, and Cardioprotective Effect of Defatted Dabai Extract. Thesis, Faculty of Medicine and Health Sciences, Universiti Putra Malaysia. Available online at: http://psasir. upm.edu.my/51137/

Khoo, H. E., Azlan, A., Ismail, A., and Abas, F. (2012). Antioxidative properties of defatted dabai pulp and peel prepared by solid phase extraction. Molecules 17, 9754-9773. doi: 10.3390/molecules17089754

Khoo, H. E., Azlan, A., Ismail, A., Abas, F., and Hamid, M. (2014). Inhibition of oxidative stress and lipid peroxidation by anthocyanins from defatted Canarium odontophyllum pericarp and peel using in vitro bioassays. PloS ONE 9:e81447. doi: 10.1371/journal.pone.0081447

Lo, S.-K., Tan, C.-P., Long, K., Yussof, M. S. A., and Lai, O.-M. (2008). Diacylglycerol oil-properties, processes and products: a review. Food Bioprocess Tech. 1, 223-233. doi: 10.1007/s11947-007-0049-3

Lv, Z. C., Yin, Y., Lin, L. J., and Peng, Y. H. (2014). Chemical constituents from Canarium pimela fruits. J. Chin. Med. Mater. 37, 1801-1803.

Marina, A. M., Man, Y. C., Nazimah, S. A. H., and Amin, I. (2009). Chemical properties of virgin coconut oil. J. Am. Oil Chem. Soc. 86, 301-307. doi: 10.1007/s11746-009-1351-1

McCarty, M. F., and DiNicolantonio, J. J. (2016). Lauric acid-rich medium-chain triglycerides can substitute for other oils in cooking applications and may have limited pathogenicity. Open Heart 3:e000467. doi: 10.1136/openhrt-2016-000467

Momchilova, S., and Nikolova-Damyanova, B. (2003). Stationary phases for silver ion chromatography of lipids: preparation and properties. J. Sep. Sci. 26, 261-270. doi: 10.1002/jssc.200390032
Mukhopadhyay, M. (2009). Extraction and processing with supercritical fluids. $J$. Chem. Technol. Biotechnol. 84, 6-12. doi: 10.1002/jctb.2072

Ntumba, J. K., Collard, L., Taba, K. M., and Robiette, R. (2015). Isolation of a series of fatty acid components of Ongokea gore seed (isano) oil and their detailed structural analysis. Lipids 50, 313-322. doi: 10.1007/s11745-014-3984-6

Prasad, K. N., Chew, L. Y., Khoo, H. E., Yang, B., Azlan, A., and Ismail, A. (2011). Carotenoids and antioxidant capacities from Canarium odontophyllum Miq. fruit. Food Chem. 124, 1549-1555. doi: 10.1016/j.foodchem.2010.08.010

Shakirin, F. H., Azlan, A., Ismail, A., Amom, Z., and Cheng Yuon, L. (2012a). Protective effect of pulp oil extracted from Canarium odontophyllum Miq. fruit on blood lipids, lipid peroxidation, and antioxidant status in healthy rabbits. Oxid. Med. Cell. Longev. 2012:840973. doi:10.1155/2012/840973

Shakirin, F. H., Azlan, A., Ismail, A., Amom, Z., and Yuon, L. C. (2012b). Antiatherosclerotic effect of Canarium odontophyllum Miq. fruit parts in rabbits fed high cholesterol diet. Evid. Based Complement. Alternat. Med. 2012:838604. doi: 10.1155/2012/838604

Siri-Tarino, P. W., Sun, Q., Hu, F. B., and Krauss, R. M. (2010). Saturated fatty acids and risk of coronary heart disease: modulation by replacement nutrients. Curr. Atheroscler. Rep. 12, 384-390. doi: 10.1007/s11883-010-0131-6

Song, C. (2015). Biosynthesis of Acylphloroglucinol Glucosides in Strawberry Fruit. Thesis, Technische Universität München. Available online at: https:// mediatum.ub.tum.de/1271772

Spiteller, G. (1998). Linoleic acid peroxidation-the dominant lipid peroxidation process in low density lipoprotein-and its relationship to chronic diseases. Chem. Phys. Lipids 95, 105-162. doi: 10.1016/S0009-3084(98)00091-7

Zock, P. L., de Vries, J. H., and Katan, M. B. (1994). Impact of myristic acid versus palmitic acid on serum lipid and lipoprotein levels in healthy women and men. Arterioscler. Thromb. 14, 567-575.

Conflict of Interest Statement: The authors declare that the research was conducted in the absence of any commercial or financial relationships that could be construed as a potential conflict of interest.

Copyright (c) 2019 Khoo, Azlan and Abd Kadir. This is an open-access article distributed under the terms of the Creative Commons Attribution License (CC BY). The use, distribution or reproduction in other forums is permitted, provided the original author(s) and the copyright owner(s) are credited and that the original publication in this journal is cited, in accordance with accepted academic practice. No use, distribution or reproduction is permitted which does not comply with these terms. 